\title{
Excitation energy transfer and equilibration process in LHCIl studied by multidimensional electronic spectroscopy
}

\author{
Thanh Nhut Do ${ }^{1}$, Adriana Huerta-Viga ${ }^{1}$, Cheng Zhang ${ }^{1}$, Parveen Akhtar ${ }^{2}$, Pawet J. \\ Nowakowski ${ }^{1}$, Muhammad Faisal bin Khyasudeen ${ }^{1}$, Hoang Long Nguyen ${ }^{1}$, Petar H. \\ Lambrev $^{2}$, and Howe-Siang Tan ${ }^{1, *}$ \\ ${ }^{1}$ School of Physical and Mathematical Sciences, Nanyang Technological University, 21 Nanyang \\ Link, Singapore 637371. \\ ${ }^{2}$ Biological Research Center, Hungarian Academy of Sciences, Temesvári körút 62, Szeged 6726, \\ Hungary.
}

\begin{abstract}
Light-harvesting complex II (LHCII) - the light-harvesting antenna of Photosystem II - is a naturally abundant system that plays an important role in photosynthesis. In this study, we present a phenomenological analysis of the excitonic energy transfer in LHCII using ultrafast two-dimensional electronic spectroscopy, that we find compares well with previous theoretical and experimental results.
\end{abstract}

\section{Introduction}

Light-harvesting complex II (LHCII) is the most abundant light-harvesting antenna in the biosphere. LHCII exists in nature mainly as a trimer, and each monomeric unit contains 8 chlorophylls $a, 6$ chlorophylls $b$ and xantophylls, pigments that are bound by three transmembrane $\alpha$ helices. The orientations of the pigments are optimized to enable ultrafast excitonic energy transfer (EET) processes that happen in the femtosecond to picosecond timescales [1].

To elucidate the ultrafast EET network in LHCII, we use two-dimensional electronic spectroscopy (2DES), implemented in a pump-probe geometry aided by an acousto-optic programmable dispersive filter [2]. 2DES with an additional spectral dimension compared to conventional transient absorption spectra is a powerful technique that is widely used to investigate ultrafast phenomena in various systems [3].

In this paper, we present a procedure to characterize the EET network in LHCII at $77 \mathrm{~K}$ through a non-linear fit model to experimental 2DES spectra. We use trimeric LHCII samples dissolved in $60 \%$ (v/v) glycerol/buffer solution. Laser pulse energies are kept low enough to avoid exciton-exciton annihilation.

\footnotetext{
* Corresponding author: howesiang@ntu.edu.sg
} 


\section{Model}

Based on previous theoretical studies [4,5], we estimate that our excitation laser spectrum covers nine excitonic states in LHCII (Fig. 1). We therefore consider nine excitonic states in our model.

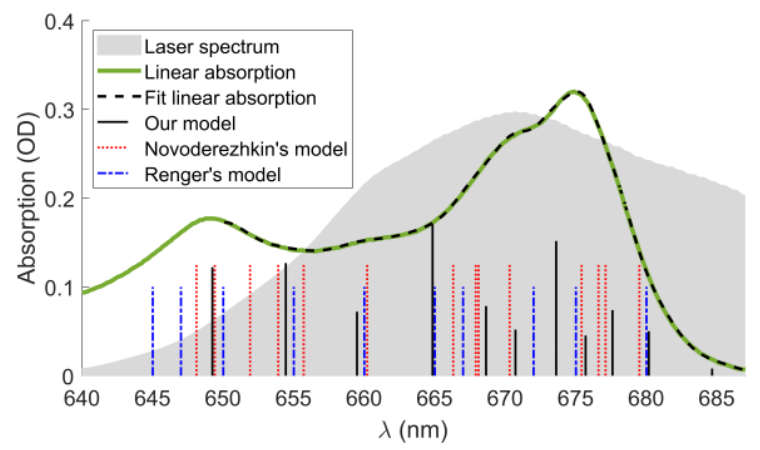

Fig. 1. Linear absorption spectrum of LHCII at $77 \mathrm{~K}$ overlaid with the fitted result (dash thick line). The excitation laser spectrum is shown with the shaded area. The vertical lines represent exciton energy levels obtained from our model (solid lines), from work by Novoderezhkin et. al [4] (dotted lines) and Renger et al. [5] (dash-dotted lines). The heights of the lines representing our model are proportional to the square of the corresponding transition dipole moments. The states at $649 \mathrm{~nm}$ and $684 \mathrm{~nm}$ are added to fit the linear spectrum and not included in the Hamiltonian.

We construct a Hamiltonian with diagonal and off-diagonal elements representing site energies and inter-site coupling, respectively [6]. We consider states up to the double quanta manifold. Diagonalization of the Hamiltonian yields the excitonic energies from which a purely absorptive 2DES spectrum before any EET processes can be simulated [7]. Simultaneously, a linear absorption spectrum can also be constructed. A transfer matrix containing fast sub-200 fs rates is then assumed to simulate a 2DES spectrum at waiting time $\mathrm{T}_{\mathrm{w}}=200 \mathrm{fs}$.

We construct a non-linear least square optimization routine to simultaneously fit to the 2DES spectrum at $\mathrm{T}_{\mathrm{w}}=200 \mathrm{fs}$ and the linear spectrum, using 2D elliptical Gaussian functions and 1D Gaussian functions, respectively, as basis. As a result, the excitonic energy levels, transition dipole moment amplitudes and linewidths can be extracted (Fig. 1 and 2).

These parameters are then kept fixed, while the amplitudes of the Gaussians (that constitutes the 2DES spectra) are allowed to float to simultaneously fit to experimental 2DES spectra measured for a whole series of $T_{w}$ from 200 fs to 100 ps. This allow us to acquire the transfer-rate matrix containing information of the EET kinetics $[8,9]$.

\section{Results and discussions}

We can see from Fig. 1 and Fig. 2 that we can fit the linear absorption spectrum and the 2DES spectrum at $\mathrm{T}_{\mathrm{w}}=200 \mathrm{fs}$, well. Our model incorporates around 10 parameters per excitonic state, and it is likely that some of the fit parameters are not unique. We found that whereas the excitonic energy levels (eigenvalues of the system Hamiltonian) can be obtained quite robustly, the fitted inter-site coupling strengths vary widely depending on the initial values of the optimization process. Additionally, we do not include in our model angles between transition dipole moments, and contributions from intraband-excited state absorptions are neglected. Improvements to the model are possible, in combination with more experimental observables like polarization dependent 2DES spectra [9]. 

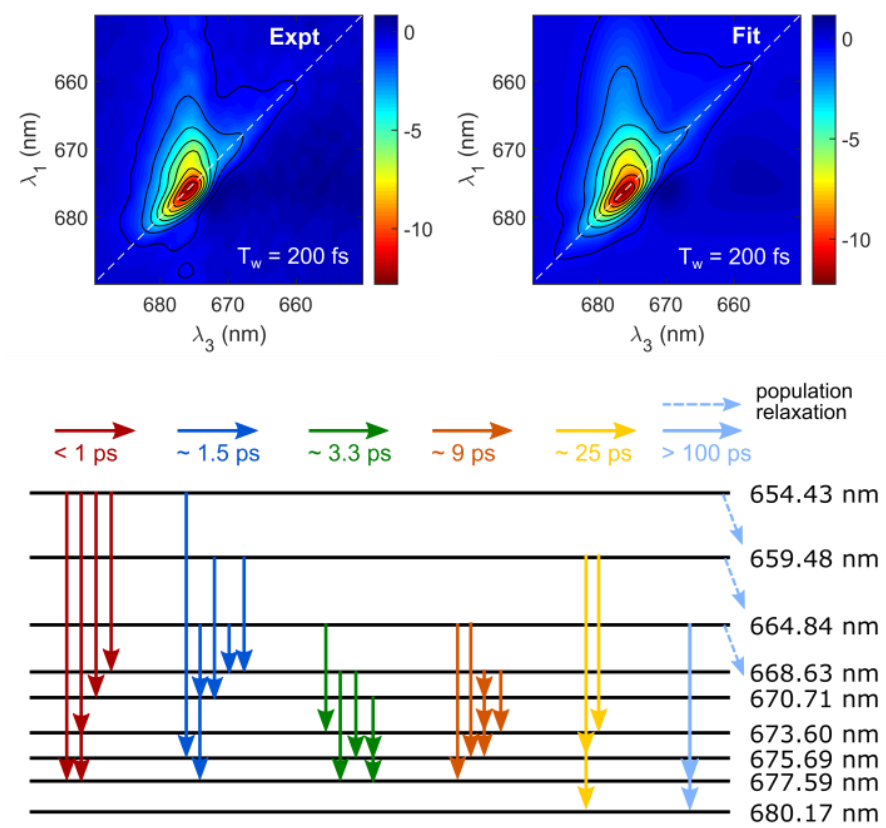

Fig. 2. Comparison between the experimental (top-left) and fitted (top-right) $2 \mathrm{DES}$ spectrum at $T_{w}=$ $200 \mathrm{fs}$. Energy levels with EET rates indicated by the arrows resulting from our fitting procedure (bottom). All rates slower than $500 \mathrm{ps}$ are omitted.

The bottom panel of Fig. 2 presents the nine excitonic energy levels and the EET rates obtained from the fit. In general, the rates that we obtain are faster than structure based theoretical model by Renger et al. [5]. A quantitative comparison should be possible after a thorough evaluation of the robustness of the fitting results and the uniqueness of the parameters.

This work was funded by the Ministry of Education Singapore (MOE2015-T2-1-039).

\section{References}

1. H. van Amerongen, R. Croce, Photosynth. Res. 116, 251 (2013).

2. K. L. Wells, P. H. Lambrev, Z. Zhang, G. Garab, H.-S. Tan, Phys. Chem. Chem. Phys. 16, 11640 (2014).

3. F. D. Fuller, J. P. Ogilvie, Annu. Rev. Phys. Chem. 66, 667 (2015).

4. V. Novoderezhkin, A. Marin, R. van Grondelle, Phys. Chem. Chem. Phys. 13, 17093 (2011).

5. T. Renger, M. E. Madjet, A. Knorr, F. Müh, J. Plant. Physiol. 168, 1497 (2011).

6. M. Cho, H. M. Vaswani, T. Brixner, J. Stenger, G. R. Fleming, J. Phys. Chem. B, 109, 10542 (2005).

7. S. Wouterson, P. Hamm, J. Phys. Chem. B, 104, 11316 (2000).

8. Z. Zhang, P. H. Lambrev, K. L. Wells, G. Garab, H.-S. Tan, Nat. Comm., 6, 7194 (2015).

9. E. Thyrhaug, K. Židek, J. Dostál, D. Bína, D. Zigmantas, J. Phys. Chem. Lett., 7, 1652 (2016). 